\title{
Comparison Between Mini PCNL and Standard PCNL in Management of Nephrolithiasis
}

\section{Bharat Bahadur Bhandari, Bikash Bikram Thapa, Dhirendra Ayer, Suresh Thapa and \\ Sanjeeb Bista}

Department of Surgery, Nepalese Army Institute of Health Sciences, Shree Birendra Hospital, Chhauni, Kathmandu, Nepal

\begin{abstract}
Introduction: The incidence of small and medium size renal stones is rising. Stone clearance, bleeding, urine leak and infectious complications are major concerns for urologist. Urologist chooses best technique from list of armamentarium available. Minimally invasive approach like Percutaneous Nephrolithotomy (PCNL) has significantly influenced the renal stone management since 1976. Miniaturisation of the instruments allow more effective and safer alternatives for urolithasis management.

Methods: This is a retrospective study in which the outcome of mini PCNL (mPCNL) was compared with standard PCNL (sPCNL) in management of nephrolithiasis.

Result: There were no significant difference in stone free rate between mPCNL and sPCNL $(96.2 \pm 3.6 \%$ vs $95.3 \pm 4.8 \%)$. The total operative time was longer in mPCNL $(55.2 \pm 19.0$ minute vs $62 \pm 21.0$ minute $)$ but the difference was not statistically significant.

Conclusions: Mini PCNL is as effective as standard PCNL with fewer bleeding complications in management of medium sized nephrolithiasis.
\end{abstract}

Key words: mini percutaneous nephrolithotomy; nephrolithiasis; percutaneous nephrolithotomy

Correspondence: Bharat Bahadur Bhandari, Department of Surgery, Nepalese Army Institute of Health Sciences, Shree Birendra Hospital, Chhauni, Kathmandu, Nepal. E-mail: drbharatbhandari@yahoo.com

DOI: http://dx.doi.org/10.3126/mjsbh.v19i2.26640

Submitted on: 2019-12-09

Accepted on: 2020-03-10

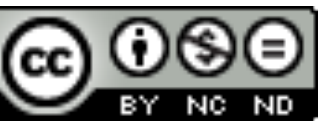

This work is licensed under creative common license:

http://creativecommons.org/licenses/by-nc-nd/4.0/ C MJSBH 2020 


\section{INTRODUCTION}

There has been increase in incidence and prevalence of nephrolithiasis globally. ${ }^{1}$ There is a paradigm shift in the management of the nephrolithiasis with the introduction of the minimally invasive endourological procedure. The international guidelines recommend Percutaneous Nephrolithotomy (PCNL) as first line of treatment for renal stones more than $20 \mathrm{~mm}$ size. $^{2}$ The procedure PCNL has evolved since 1976 and has undergone many modifications. One of them is miniaturising the access sheath. Standard PCNL (sPCNL) is done with sheath size of 24-30 French (Fr) where as the Mini PCNL/Miniperc is done with sheath size 14 - 20 Fr. Mini PCNL (mPCNL) is safer and had equal efficacy rate for management of renal stones. ${ }^{3}$ We evaluate and compare the mini PCNL and standard PCNL in terms of outcome and stone free rate.

\section{METHODS}

The hospital records of patients who underwent PCNL (December 2015 till August 2018) was reviewed in this retrospective study. The records showed 95 patients underwent standard PCNL (sPCNL) and 79 patients underwent mini PCNL (mPCNL). The data were collected on stone details and patient characteristics. The post procedural data collected were total operative time, drop in haemoglobin, infectious and bleeding complications, post operative analgesic use, hospital stay, urine leak and stone free rate. The data collected were analysed and compared between standard min-PCNL and tubeless PCNL. Data were compiled in Medcal $^{R}$ and statistical analysis done. Student $t$ test and Chi square test were done to compare summary measures of stone details and patient characteristics. $\mathrm{P}$ value of $<0.05$ was considered statistically significant.

\section{RESULTS}

The preoperative diagnosis of nephrolithiasis and the stone burden was done by Non-Contrast Tomography KUB in all cases (95 sPCNL and 79 mPCNL). Urine sterility was ensured before undertaking procedure. All patients underwent prone PCNL under general anaesthesia after single dose intravenous third generation cephalosporin. The calyceal puncture was done under fluoroscopy guidance after retrograde injection of diluted contrast through 5/6 Fr ureteric catheter (1:3), with $18 \mathrm{G}$ needle by triangulation method. After parking safety guidewire $0.38 " / 150 \mathrm{~cm}$ tract was dilated with serial Teflon fascial dilators or coaxial metallic dilator. The procedure was continued using access sheath of size $30 \mathrm{Fr}$ in sPCNL and 18 to 20 Fr in mPCNL. Stone localisation was done with 24 Fr nephroscope in sPCNL and with 9.5/11.5 Fr, ureteroscope (Karl Storz, Germany). The stone was fragmented with pneumatic lithotripsy in both groups. Irrigation pump was used and stone fragments were removed with alligator forceps in sPCNL. During mPCNL the stone fragments were largely retrieved with Bernoulli manoeuvre and stone holding forceps. The decision to omit nephrostomy tube was under surgeon's discretion depending up on the stone clearance, intraoperative bleeding, and urine colour. In tubeless PCNL (18 sPCNL and $33 \mathrm{mPCNL}$ ) only the indwelling ureteric stent (DJ stent) $26 \mathrm{~cm} / 6 \mathrm{Fr}$ was kept. Total tubeless PCNL was done in 14 out of $33 \mathrm{mPCNL}$ where the ureteric catheter was left in situ and removed on first postoperative day after confirming absence of residual stone in urinary tract. Stone free rate was accessed with on table fluoroscopy and postoperative x-ray KUB and USG KUB if necessary.

The mean age of patients was $36.8 \pm 9.1$ and $34.8 \pm$ 7.4 years in sPCNL and mPCNL respectively. The patient characteristics and stone characteristics in both groups were comparable (table 1) with mean stone size of $22.8 \pm 2.9 \mathrm{~mm}$ in sPCNL and $20.8 \pm$ $3.5 \mathrm{~mm}$ in mPCNL group. There was no significant difference in total post operative time, overall stone free rate $(96.2 \pm 3.6 \%$ vs $95.3 \pm 4.8 \%)$ and postoperative complications and total hospital stay in both group (table 2). Post operative drain site leak was higher in sPCNL group. The drop in post operative haemoglobin $(3.3 \pm 0.5 \mathrm{gm} \%$ vs $1.3 \pm 0.8$ gm\%) was significantly less in mPCNL than sPCNL $(\mathrm{p}<0.05)$. In subgroup analysis the total stone free rate was higher in $\mathrm{mPCNL}$ for multiple and calyceal stones. Significantly greater number of patient underwent tubeless PCNL in mini PCNL group than standard PCNL (18.9\% vs 39\%). The percentage of auxiliary procedure was higher in mPCNL group (7.5\%). Three patients underwent 
Table 1. Patient and stone characteristics

\begin{tabular}{|c|c|c|c|}
\hline & sPCNL & mPCNL & p value \\
\hline $\mathbf{N}$ & 95 & 79 & \\
\hline Mean Age (years) & $\begin{array}{r}36.8 \pm \\
9.1\end{array}$ & $\begin{array}{r}34.8 \pm \\
7.4\end{array}$ & NS \\
\hline $\begin{array}{l}\text { Mean Stone Size } \\
(\mathrm{mm})\end{array}$ & $\begin{array}{r}22.8 \pm \\
2.9\end{array}$ & $\begin{array}{r}20.8 \pm \\
3.5\end{array}$ & NS \\
\hline M:F & $3.1: 1$ & 2.8:1 & \\
\hline Laterality & $\begin{array}{r}\text { Right- } 43 \\
\text { left- } 52\end{array}$ & $\begin{array}{r}\text { Right- } 36 \\
\text { left- } 43\end{array}$ & \\
\hline Stone location (n) & & & NS \\
\hline Renal pelvis & 28 & 26 & \\
\hline Calyceal & 67 & 53 & \\
\hline Stone number (n) & & & NS \\
\hline Single & 45 & 30 & \\
\hline Multiple & 50 & 49 & \\
\hline $\begin{array}{l}\text { Recurrent } \\
\text { Disease }\end{array}$ & 5 & 2 & \\
\hline
\end{tabular}

ureterorenoscopic lithotripsy for steinstrasse in mPCNL group. Three patients underwent shock wave lithotripsy in each group. Two patients in sPCNL and three patients in mPCNL underwent second session PCNL for residual stones.

\section{DISCUSSION}

Jackman performed the first Mini PCNL in adult, using small access sheath (13 Fr) with miniature instrument in 1997. ${ }^{4}$ The very first Mini PCNL in seven adult patients had encouraging result with stone free rate of $89 \%$. Li et al. published reports with comparable stone free rate between mini and standard PCNL with significantly lesser rate of blood transfusion in mini PCNL group $(1.1 \%$ vs. $6.9 \%){ }^{5}$ Mini PCNL is associated with higher number of tubeless PCNL, lesser hospital stay (3.2 vs. 4.8 days, $\mathrm{p}<0.05)$ and reduced drop in haemoglobin $(0.8 \pm 0.9$ vs. $1.3 \pm 0.4 \mathrm{gm} \%, \mathrm{P}=$ 0.01). ${ }^{6}$ The higher drop in haemoglobin in our series is due to use of coaxial metallic dilators and inclusion of recurrent nephrolithasis (post open surgery). $50 \%$ to $95 \%$ of mini PCNL were done tubeless worldwide with similar outcomes. Literature shows no statistically significant difference in total operative time between mini PCNL and standard PCNL (24 - 155 minutes Vs 25
Table 2. Comparison of Postoperative outcomes

\begin{tabular}{|c|c|c|c|}
\hline & sPCNL & mPCNL & $\begin{array}{c}\mathbf{p} \\
\text { value }\end{array}$ \\
\hline No of puncture & 95 & 79 & \\
\hline Teflon serial & 59 & 56 & \\
\hline Coaxial metallic & 36 & 23 & \\
\hline $\begin{array}{l}\text { Operative time } \\
\text { (minute) }\end{array}$ & $55.2 \pm 19.0$ & $62 \pm 21.0$ & NS \\
\hline $\begin{array}{l}\text { Post op Hb drop } \\
(\text { Gm\%) }\end{array}$ & $3.3 \pm 0.5$ & $1.3 \pm 0.8$ & 0.01 \\
\hline Post op leakage & 5 & 1 & 0.04 \\
\hline Tubeless PCNL & 20 & 31 & 0.01 \\
\hline Hospital stay & $3 \pm 1.2$ & $2.2 \pm 1.3$ & NS \\
\hline SFR (\%) overall & & & \\
\hline single & $96.2 \pm 3.6$ & $95.3 \pm 4.8$ & NS \\
\hline multiple & $94 \pm 2.6$ & $97.3 \pm 4.8$ & \\
\hline calyceal & $93 \pm 2.1$ & $97.8 \pm 3.4$ & \\
\hline $\begin{array}{l}\text { Auxillary } \\
\text { procedure (n) }\end{array}$ & 3 & 6 & NS \\
\hline $\begin{array}{l}\text { Complications } \\
(\%)\end{array}$ & $19(24.2$ & 14 & NS \\
\hline Grade I & $18 \%$ & $16.2 \%$ & \\
\hline Grade II & $2.8 \%$ & $3.2 \%$ & \\
\hline Grade III & $3.6 \%$ & $2.6 \%$ & \\
\hline
\end{tabular}

- 103 minutes). The reported stone free rate (SFR) for stone burden $(10-30 \mathrm{~mm})$ was $88 \%-96 \%$ in mini PCNL and $95-100 \%$ in standard PCNL. ${ }^{6-8}$

Complex stone burden with stone size of 10 to 35 $\mathrm{mm}$ can be effectively managed with minimum blood loss in Mini-PCNL technique. Elsheemy et al. ${ }^{9}$ randomised stones ( irrespective of size and location into sPCNL or mPCNL ( $\mathrm{n}=151$ vs. 378$)$. $\mathrm{He}$ found that mini-PCNL has longer operative times, shorter hospital stay and higher rate of tubeless PCNL with lesser complication rate $(7.9 \%$ vs $20.5 \%$ ). Complex stone burden had lesser overall SFR during mPCNL ( $86.8 \%$ vs. $90.7 \%$ in first session) and required multiple tracts or multiple sessions of PCNL. ${ }^{8,9}$ Postoperative pain and fever, bleeding, urine leak were common complications in both group. Tubeless mini PCNL requires less pain medication.,10,11 Zeng G. et al. ${ }^{10}$ claimed that the overall complication rate after mini PCNL $(n=10,000)$ was $20.1 \%$, out of which 
$7.4 \%$ are Clavien grade I, $8.8 \%$ was grade II and $3.5 \%$ was grade III complications, but no grade IV or V complications (Zeng). SFR was higher with simple with low burden nephrolithiasis. Multiple calyceal stones were more efficiently managed with mPCNL. 8,12

This study has some limitations. This is a retrospective study done in a single centre, with limited number of patients.

\section{CONCLUSIONS}

Mini PCNL is as effective as standard PCNL with less blood loss in small and medium size stone (10 to $30 \mathrm{~mm}$ ). Stone burden is the key factor for optimal stone free rate. Higher number of tubeless procedure can be performed with lesser morbidity. This study is retrospective and comprises small sample size. Puncture technique and energy used for lithotripsy can act as significant confounders in outcome.

To cite this article: Bhandari BB, Thapa BB, Ayer D, Thapa S, Bista S. Comparison between Mini PCNL and Standard PCNL in management of Nephrolithiasis. MJSBH. 2020;19(2):80-3.

Conflict of Interest: None declared

\section{REFERENCES}

1. Romero V, Akpinar H, Assimos DG. Kidney stones: a global picture of prevalence, incidence, and associated risk factors. Rev Urol. 2010;12(2-3):e86. PMID: 20811557

2. Türk C, Knoll T, Petrik A, Sarica K, Straub M, Seitz C. Guidelines on Urolithiasis. European Association of Urology. 2019. Available from: https://uroweb.org/wp-content/uploads/18_Urolithiasis.pdf

3. Wright A, Rukin N, Smith D, De la Rosette J, Somani BK. 'Mini, ultra, micro' - nomenclature and cost of these new minimally invasive percutaneous nephrolithotomy (PCNL) techniques. Ther Adv Urol. 2016;8(2)142-6. doi: $10.1177 / 1756287215617674$

4. Jackman SV, Docimo SG, Cadeddu JA, Bishoff JT, Kavoussi LR, Jarrett TW. The "mini-perc" technique: a less invasive alternative to percutaneous nephrolithotomy. World J Urol. 1998;16(6):371-4. PMID:9870281

5. Li LY, Gao X, Yang M, Li JF, Zhang HB, Xu WF, et al. Does a smaller tract in percutaneous nephrolithotomy contribute to less invasiveness? A prospective comparative study. Urology. 2010;75(1). PMID:19800671

6. Mishra S, Sharma R, Garg C, Kurien A, Sabnis R, Desai M. Prospective comparative study of miniperc and standard PNL for treatment of 1 to $2 \mathrm{~cm}$ size renal stone. BJU Int. 2011;108(6). PMID: 21477212

7. Kukreja RA. Should mini percutaneous nephrolithotomy (MiniPNL/Miniperc) be the ideal tract for medium-sized renal calculi (15-30 mm)? World J Urol. 2018;36(2)285-91. PMID:29134277

8. Cheng F, Yu W, Zhang X, Yang S, Xia Y, Ruan Y. Minimally invasive tract in percutaneous nephrolithotomy for renal stones. J Endourol. 2010;24(10):1579-82. PMID:20839954

9. ElSheemy MS, Elmarakbi AA, Hytham M, Ibrahim H, Khadgi S, Al-Kandari AM. Mini vs standard percutaneous nephrolithotomy for renal stones: a comparative study. Urolithiasis. 2018;16(10)207-14. PMID: 29549382

10. Zeng G, Zhao Z, Wan S, Mai Z, Wu W, Zhong W, et al. Minimally invasive percutaneous nephrolithotomy for simple and complex renal caliceal stones: a comparative analysis of more than 10,000 cases. J Endourol. 2013;27(10):1203-8. PMID:23924320

11. Hu G, Guo Z, Liu H, Luo M, Liu M, Lai P, et al. A novel minimally invasive percutaneous nephrolithotomy technique: safety and efficacy report. Scand J Urol. 2015;49(2):174-80. PMID:25247463

12. Ganesamoni R, Sabnis RB, Mishra S, Parekh N, Ganpule A, Vyas JB, et al. Prospective randomised controlled trial comparing laser lithotripsy with pneumatic lithotripsy in miniperc for renal calculi. J Endourol. 2013;27(12). 1444-9. PMID:24251428 